\title{
Cefamandole Sodium
}

National Cancer Institute

\section{Source}

National Cancer Institute. Cefamandole Sodium. NCI Thesaurus. Code C47967.

The sodium salt form of cefamandole, a beta-lactam, second-generation cephalosporin antibiotic with bactericidal activity. Cefamandole binds to and inactivates penicillin-binding proteins (PBP) located on the inner membrane of the bacterial cell wall. Inactivation of PBPs interferes with the cross-linkage of peptidoglycan chains necessary for bacterial cell wall strength and rigidity. This results in the weakening of the bacterial cell wall and causes cell lysis. 